\title{
Mann Type Iteration Method Involving Three Strictly Hemicontractive Mappings in Banach Spaces
}

\author{
B. S. Lee ${ }^{1, *}$, Arif Rafiq ${ }^{2}$ \\ ${ }^{1}$ Department of Mathematics, Kyungsung University, Busan 608-736, Korea \\ ${ }^{2}$ Department of Mathematics, Lahore Leads University, Lahore, Pakistan \\ ${ }^{*}$ Corresponding Author: bslee@ks.ac.kr
}

Copyright (C2013 Horizon Research Publishing All rights reserved.

\begin{abstract}
The purpose of this paper is to prove that the modified Mann iteration process can be applied to approximate the common fixed point of three strictly hemicontractive mappings in smooth Banach spaces.
\end{abstract}

Keywords Mann type iteration method, Strictly hemicontractive mappings, Continuous mappings, Lipschitz mappings, Smooth Banach spaces

AMS Subject Classification: Primary 47H10, 47H17, Secondary 54H25

\section{Introduction}

Let $K$ be a nonempty subset of an arbitrary Banach space $X$ and $X^{*}$ be its dual space. The symbols $D(T)$, $R(T)$ and $F(T)$ stand for the domain, the range and the set of fixed points of a mapping $T: X \rightarrow X$, respectively. We denote by $J$ the normalized duality mapping from $X$ to $2^{X^{*}}$ defined by

$$
J(x)=\left\{f^{*} \in X^{*}:\left\langle x, f^{*}\right\rangle=\|x\|^{2}=\left\|f^{*}\right\|^{2}\right\},
$$

where $\langle.,$.$\rangle denotes the duality pairing. In a smooth Banach space J$ is single-valued (and denoted by $j$ ).

Remark 1.1 1. $X$ is said to be uniformly smooth if $X^{*}$ is uniformly convex.

2. In a uniformly smooth Banach space, $J$ is uniformly continuous on bounded subsets of $X$.

Let $T$ be a self-mapping of $K$.

Definition $1.1 T$ is said to be Lipshitzian if there exists $L>0$ such that

$$
\|T x-T y\| \leqslant L\|x-y\|,
$$

for all $x, y \in K$. If $L=1$, then $T$ is said to be non-expansive and if $0 \leqslant L<1$, then $T$ is said to be contractive.

Definition 1.2 [ 3, 5]

1. $T$ is said to be pseudocontractive if the inequality

$$
\|x-y\| \leqslant \| x-y+t((I-T) x-(I-T) y \|,
$$

holds for each $x, y \in K$ and for all $t>0$.

2. $T$ is said to be strongly pseudocontractive if there exists $t>1$ such that

$$
\|x-y\| \leq\|(1+r)(x-y)-r t(T x-T y)\|
$$

for all $x, y \in D(T)$ and $r>0$. 
3. $T$ is said to be local strongly pseudocontractive if for each $x \in D(T)$ there exists $t_{x}>1$ such that

$$
\|x-y\| \leq\left\|(1+r)(x-y)-r t_{x}(T x-T y)\right\|
$$

for all $y \in D(T)$ and $r>0$.

4. $T$ is said to be strictly hemicontractive if $F(T) \neq \emptyset$ and if there exists $t>1$ such that

$$
\|x-q\| \leq\|(1+r)(x-q)-r t(T x-q)\|
$$

for all $x \in D(T), q \in F(T)$ and $r>0$.

Clearly, a strongly pseudocontractive mapping is local strongly pseudocontractive.

Chidume [3] established that the Mann iteration sequence converges strongly to the unique fixed point of $T$ in case $T$ is a Lipschitz strongly pseudo-contractive mapping from a bounded closed convex subset of $L_{p}$ (or $l_{p}$ ) into itself. Schu [13] generalized the result in [3] to both uniformly continuous strongly pseudo-contractive mappings and real smooth Banach spaces. Park [10] extended the result in [3] to both strongly pseudocontractive mappings and certain smooth Banach spaces. Rhoades [11] proved that the Mann and Ishikawa iteration methods may exhibit different behaviors for different classes of nonlinear mappings. Afterwards, several generalizations have been made in various directions (see for examples $[4,7-8,10,14]$ ).

Let $K$ be a nonempty closed bounded convex subset of an arbitrary smooth Banach space $X$ and $T, S, H: K \rightarrow$ $K$ be three continuous strictly hemicontractive mappings. We proved that the sequence generated by the Mann type iteration method converges strongly to the common fixed point of $T, S$ and $H$. 13-14].

The results presented in this paper extend and improve the corresponding results, particularly in [3-4, 8-10,

\section{Preliminaries}

We need the following results.

Lemma 2.1 [10] Let $X$ be a smooth Banach space. Suppose that one of the following holds:

(1) $J$ is uniformly continuous on any bounded subsets of $X$,

(2) $\langle x-y, j(x)-j(y)\rangle \leq\|x-y\|^{2}$, for all $x, y$ in $X$,

(3) for any bounded subset $D$ of $X$, there is a functional $g:[0, \infty) \rightarrow[0, \infty)$ such that

$$
\begin{aligned}
\operatorname{Re}\langle x-y, j(x)-j(y)\rangle & \leq g(\|x-y\|), \text { for all } x, y \in D \text {, where } g \text { satisfies } \\
\lim _{t \rightarrow 0^{+}} \frac{g(t)}{t} & =0 .
\end{aligned}
$$

Then for any $\epsilon>0$ and any bounded subset $K$, there exists $\delta>0$ such that

$$
\|s x+(1-s) y\|^{2} \leq(1-2 s)\|y\|^{2}+2 s \operatorname{Re}\langle x, j(y)\rangle+2 s \epsilon
$$

for all $x, y \in K$ and $s \in[0, \delta]$.

Remark 2.1 1. If $X$ is uniformly smooth, then (1) in Lemma 2.1 holds.

2. If $X$ is a Hilbert space, then (2) in Lemma 2.1 holds.

Lemma 2.2 [4] Let $T: D(T) \subseteq X \rightarrow X$ be a mapping with $F(T) \neq \emptyset$. Then $T$ is strictly hemicontractive if and only if there exists $t>1$ such that for all $x \in D(T)$ and $q \in F(T)$, there exists $j(x-q) \in J(x-q)$ satisfying

$$
\operatorname{Re}\langle x-T x, j(x-q)\rangle \geq\left(1-\frac{1}{t}\right)\|x-q\|^{2} .
$$

Lemma 2.3 [8] Let $X$ be an arbitrary normed linear space and $T: D(T) \subseteq X \rightarrow X$ be a mapping.

(1) If $T$ is local strongly pseudocontractive and $F(T) \neq \emptyset$, then $F(T)$ is a singleton and $T$ is strictly hemicontractive.

(2) If $T$ is strictly hemicontractive, then $F(T)$ is a singleton.

Lemma 2.4 [8] Let $\left\{\theta_{n}\right\}_{n=0}^{\infty},\left\{\xi_{n}\right\}_{n=0}^{\infty}$ and $\left\{\eta_{n}\right\}_{n=0}^{\infty}$ be nonnegative real sequences and let $\epsilon^{\prime}>0$ be a constant satisfying

$$
\xi_{n+1} \leq\left(1-\theta_{n}\right) \xi_{n}+\epsilon^{\prime} \theta_{n}+\eta_{n}, n \geq 0
$$

where $\sum_{n=0}^{\infty} \theta_{n}=\infty, \theta_{n} \leq 1$ for all $n \geq 0$ and $\sum_{n=0}^{\infty} \eta_{n}<\infty$.

Then, $\lim _{n \rightarrow \infty} \sup \xi_{n} \leq \epsilon^{\prime}$. 


\section{Main Results}

We now prove our main results.

Theorem 3.1 Let $X$ be a smooth Banach space satisfying any of the Axioms (1)-(3) of Lemma 2.1. Let K be a nonempty closed bounded convex subset of $X$ and $T, S, H: K \rightarrow K$ be three continuous strictly hemicontractive mappings. Let $\left\{\alpha_{n}\right\}_{n=0}^{\infty},\left\{\beta_{n}\right\}_{n=0}^{\infty},\left\{\gamma_{n}\right\}_{n=0}^{\infty}$ and $\left\{\delta_{n}\right\}_{n=0}^{\infty}$ be real sequences in $[0,1]$ such that $\alpha_{n}+\beta_{n}+\gamma_{n}+\delta_{n}=1$ and satisfying conditions (i) $\sum_{n=0}^{\infty}\left(1-\alpha_{n}\right)=\infty$, and (ii) $\lim _{n \rightarrow \infty}\left(1-\alpha_{n}\right)=0$.

Suppose that $\left\{x_{n}\right\}_{n=0}^{\infty}$ is the sequence generated from an arbitrary $x_{0} \in K$ by

$$
x_{n+1}=\alpha_{n} x_{n}+\beta_{n} T x_{n}+\gamma_{n} S x_{n}+\delta_{n} H x_{n}, n \geq 0 .
$$

Then the sequence $\left\{x_{n}\right\}_{n=0}^{\infty}$ converges strongly to the common fixed point $q$ of $T, S$ and $H$.

Proof By [5, Corollary 1], T, $S$ and $H$ have the unique fixed point $q$ in $K$. It follows from Lemma 2.3 that $F(T) \cap F(S) \cap F(H)$ is a singleton. That is, $F(T) \cap F(S) \cap F(H)=\{q\}$ for some $q \in K$. Now for $k=\frac{1}{t}$, where $t$ satisfies $(2.2)$.

On the other hand, for given any $\epsilon>0$ and the bounded subset $K$, there exists a $\delta>0$ satisfying (2.1). Note that the condition $(i i)$ ensures that there exists an $N$ such that

$$
1-\alpha_{n}<\min \left\{\delta, \frac{1}{2(1-k)}\right\}, n \geq N .
$$

Using (3.1) and Lemma 2.2, we infer that

$$
\begin{aligned}
\left\|x_{n+1}-q\right\|^{2}= & \left\|\alpha_{n} x_{n}+\beta_{n} T x_{n}+\gamma_{n} S x_{n}+\delta_{n} H x_{n}-q\right\|^{2} \\
= & \left\|\alpha_{n}\left(x_{n}-q\right)+\beta_{n}\left(T x_{n}-q\right)+\gamma_{n}\left(S x_{n}-q\right)+\delta_{n}\left(H x_{n}-q\right)\right\|^{2} \\
= & \| \alpha_{n}\left(x_{n}-q\right) \\
& +\left(1-\alpha_{n}\right)\left(\frac{\beta_{n}}{1-\alpha_{n}}\left(T x_{n}-q\right)+\frac{\gamma_{n}}{1-\alpha_{n}}\left(S x_{n}-q\right)+\frac{\delta_{n}}{1-\alpha_{n}}\left(H x_{n}-q\right)\right) \|^{2} .
\end{aligned}
$$

Put $d_{n}=\beta_{n}+\gamma_{n}+\delta_{n}$, then $\alpha_{n}=1-d_{n}(n \geq 0)$, then

$$
\begin{aligned}
\left\|x_{n+1}-q\right\|^{2}= & \left\|\alpha_{n}\left(x_{n}-q\right)+\left(1-\alpha_{n}\right) Y_{n}\right\|^{2} \\
= & \left\|d_{n} Y_{n}+\left(1-d_{n}\right)\left(x_{n}-q\right)\right\|^{2} \\
\leq & \left(1-2 d_{n}\right)\left\|x_{n}-q\right\|^{2}+2 d_{n} \operatorname{Re}\left\langle Y_{n}, j\left(x_{n}-q\right)\right\rangle+2 \epsilon d_{n} \\
= & \left(1-2 d_{n}\right)\left\|x_{n}-q\right\|^{2}+2 \beta_{n} \operatorname{Re}\left\langle T x_{n}-q, j\left(x_{n}-q\right)\right\rangle \\
& +2 \gamma_{n} \operatorname{Re}\left\langle S x_{n}-q, j\left(x_{n}-q\right)\right\rangle+2 \delta_{n} \operatorname{Re}\left\langle H x_{n}-q, j\left(x_{n}-q\right)\right\rangle+2 \epsilon d_{n} \\
\leq & \left(1-2 d_{n}\right)\left\|x_{n}-q\right\|^{2}+2 \beta_{n} \cdot k\left\|x_{n}-q\right\|^{2}+2 \gamma_{n} \cdot k\left\|x_{n}-q\right\|^{2} \\
& +2 \delta_{n} \cdot k\left\|x_{n}-q\right\|^{2}+2 \epsilon d_{n} \\
= & \left(1-2(1-k) d_{n}\right)\left\|x_{n}-q\right\|^{2}+2 \epsilon d_{n}
\end{aligned}
$$

for all $n \geq N$, where

$$
Y_{n}=\frac{\beta_{n}}{1-\alpha_{n}}\left(T x_{n}-q\right)+\frac{\gamma_{n}}{1-\alpha_{n}}\left(S x_{n}-q\right)+\frac{\delta_{n}}{1-\alpha_{n}}\left(H x_{n}-q\right) .
$$

Putting

$\theta_{n}=2(1-k) d_{n}, \xi_{n}=\left\|x_{n}-q\right\|, \epsilon^{\prime}=\frac{2 \epsilon}{2(1-k)}$ and $\eta_{n}=0$, and applying Lemma 2.4

we have

$$
\xi_{n+1} \leq\left(1-\theta_{n}\right) \xi_{n}+\epsilon^{\prime} \theta_{n}+\eta_{n}, n \geq 1 .
$$

Observe that $\sum_{n=0}^{\infty} \theta_{n}=\infty, \theta_{n}<1$ for all $n \geq 1$. It follows from Lemma 2.4 that

$$
\lim _{n \rightarrow \infty} \sup \left\|x_{n}-q\right\|^{2} \leq \epsilon^{\prime} .
$$

Letting $\epsilon^{\prime} \rightarrow 0^{+}$, we obtain that $\lim _{n \rightarrow \infty} \sup \left\|x_{n}-q\right\|^{2}=0$, which implies that $x_{n} \rightarrow q$ as $n \rightarrow \infty$.

Corollary 3.2 Let $X$ be a smooth Banach space satisfying any of the Axioms (1)-(3) of Lemma 2.3. Let K be a nonempty closed bounded convex subset of $X$ and $T, S, H: K \rightarrow K$ be three Lipschitz strictly hemicontractive mappings. Let $\left\{\alpha_{n}\right\}_{n=0}^{\infty},\left\{\beta_{n}\right\}_{n=0}^{\infty},\left\{\gamma_{n}\right\}_{n=0}^{\infty}$ and $\left\{\delta_{n}\right\}_{n=0}^{\infty}$ be real sequences in $[0,1]$ such that $\alpha_{n}+\beta_{n}+\gamma_{n}+\delta_{n}=1$ and satisfying conditions (i) $\sum_{n=0}^{\infty}\left(1-\alpha_{n}\right)=\infty$, and (ii) $\lim _{n \rightarrow \infty}\left(1-\alpha_{n}\right)=0$.

From arbitrary $x_{0} \in K$, define the sequence $\left\{x_{n}\right\}$ generated by the iteration process (3.1). Then the sequence $\left\{x_{n}\right\}_{n=0}^{\infty}$ converges strongly to the common fixed point $q$ of $T, S$ and $H$. 
Corollary 3.3 Let $X$ be a smooth Banach space satisfying any of the Axioms (1)-(3) of Lemma 2.3. Let K be a nonempty closed bounded convex subset of $X$ and $T: K \rightarrow K$ be a continuous strictly hemicontractive mapping. Suppose that $\left\{\alpha_{n}\right\}_{n=0}^{\infty}$ be a sequence in [0,1] satisfying conditions $(i) \lim _{n \rightarrow \infty} \alpha_{n}=0$ and $(i i) \sum_{n=0}^{\infty} \alpha_{n}=\infty$.

From arbitrary $x_{0} \in K$, define the sequence $\left\{x_{n}\right\}$ by the following Mann iteration scheme

$$
x_{n+1}=\left(1-\alpha_{n}\right) x_{n}+\alpha_{n} T x_{n}, n \geq 0 .
$$

Then the sequence $\left\{x_{n}\right\}_{n=0}^{\infty}$ converges strongly to a unique fixed point $q$ of $T$.

Corollary 3.4 Let $X$ be a smooth Banach space satisfying any of the Axioms (1)-(3) of Lemma 2.3. Let K be a nonempty closed bounded convex subset of $X$ and $T: K \rightarrow K$ be a Lipschitz strictly hemicontractive mapping. Suppose that $\left\{\alpha_{n}\right\}_{n=0}^{\infty}$ be a sequence in $[0,1]$ satisfying conditions $(i) \lim _{n \rightarrow \infty} \alpha_{n}=0$ and $(i i) \sum_{n=0}^{\infty} \alpha_{n}=\infty$.

From arbitrary $x_{0} \in K$, define the sequence $\left\{x_{n}\right\}$ by the following Mann iteration scheme

$$
x_{n+1}=\left(1-\alpha_{n}\right) x_{n}+\alpha_{n} T x_{n}, n \geq 0 .
$$

Then the sequence $\left\{x_{n}\right\}_{n=0}^{\infty}$ converges strongly to a unique fixed point $q$ of $T$.

Remark 3.1 Similar results can be found for the iteration processes involved error terms, we omit the details.

\section{Acknowledgements}

The authors thank the referees for their valuable suggestions and comments.

\section{REFERENCES}

[1] S. Chang, Some problems and results in the study of nonlinear analysis, Nonlinear Anal. TMA 30 (7) (1997), $4197-4208$.

[2] S. S. Chang, Y. J. Cho, B. S. Lee, S. M. Kang, Iterative approximations of fixed points and solutions for strongly accretive and strongly pseudocontractive mappings in Banach spaces, J. Math. Anal. Appl. 224 (1998), $149-165$.

[3] C. E. Chidume, Iterative approximation of fixed points of Lipschitzian strictly pseudocontractive mappings, Proc. Amer. Math. Soc. 99 (2) (1987), 283-288.

[4] C. E. Chidume, M. O. Osilike, Fixed point iterations for strictly hemicontractive maps in uniformly smooth Banach spaces, Numer. Funct. Anal. Optimiz. 15 (1994), 779-790.

[5] K. Deimling, Zeros of accretive operators, Manuscripta Math. 13 (1974), 365-374.

[6] S. Ishikawa, Fixed points by a new iteration method, Proc. Amer. Math. Soc. 44 (1974), 147-150.

[7] Z. Liu and S. M. Kang, Stability of Ishikawa iteration methods with errors for strong pseudocontractions and nonlinear equations involving accretive operators in arbitrary real Banach spaces, Math. and Computer Modell. 34 (2001), 319330 .

[8] Z. Liu, S. M. Kang, S. H. Shim, Almost stability of the Mann iteration method with errors for strictly hemicontractive operators in smooth Banach spaces, J. Korean Math. Soc. 40 (1) (2003), 29-40.

[9] W. R. Mann, Mean value methods in iteration, Proc. Amer. Math. Soc. 4 (1953), 506-510.

[10] J. A. Park, Mann iteration process for the fixed point of strictly pseudocontractive mapping in some Banach spaces, J. Korean Math. Soc. 31 (1994), 333-337.

[11] B. E. Rhoades, Comments on two fixed point iteration methods, J. Math. Anal. Appl. 56 (1976), 741-750.

[12] S. Reich, Constructive techniques for accretive and monotone operators, Applied Nonlinear Analysis, Academic Press (1979), 335-345.

[13] J. Schu, Iterative construction of fixed points of strictly pseudocontractive mappings, Applicable Anal. 40 (1991), 67-72.

[14] X. Weng, Fixed point iteration for local strictly pseudo-contractive mapping, Proc. Amer. Math. Soc. 113 (1991), no. 3, 727-731. 